Revue d'histoire de l'Amérique française

ZWB REVUE D.HISTOIRE DE L'AMÉRIQUE FRANÇAISE

\title{
Projets de préfectures apostoliques pour le Nord-Est du Canada
}

\section{Gaston Carrière}

Volume 17, numéro 2, septembre 1963

URI : https://id.erudit.org/iderudit/302275ar

DOI : https://doi.org/10.7202/302275ar

Aller au sommaire du numéro

Éditeur(s)

Institut d'histoire de l'Amérique française

ISSN

0035-2357 (imprimé)

1492-1383 (numérique)

Découvrir la revue

Citer cet article

Carrière, G. (1963). Projets de préfectures apostoliques pour le Nord-Est du

Canada. Revue d'histoire de l'Amérique française, 17(2), 185-212.

https://doi.org/10.7202/302275ar d'utilisation que vous pouvez consulter en ligne.

https://apropos.erudit.org/fr/usagers/politique-dutilisation/ 


\section{PROJETS DE PRÉFECTURES APOSTOLIQUES POUR LE NORD.EST DU CANADA *}

Les Oblats qui avaient réussi, en 1868, à faire retarder l'érection d'un vicariat apostolique pour le Nord-Est du Canada, pouvaient penser que la question était définitivement réglée. Les évêques cependant, et surtout celui de Rimouski, ne désarmeraient pas aussi vite et ils entreprendraient bientôt de nouvelles négociations pour atteindre le but qu'ils s'étaient proposé.

Le 11 janvier 1869, Mgr Jean Langevin, évêque de Rimouski, s'adressait directement au cardinal Barnabo et lui rappelait qu'au concile de Québec, les évêques avaient supplié le pape d'ériger un vicariat apostolique pour la région centrale de la baie d'Hudson. On disait également, affirmait Mgr Langevin, que le futur évêque aurait la charge de tous les Indiens jusqu'au Labrador, tant de ceux du diocèse de Havre-de-Grâce que de ceux qui habitaient le diocèse de Saint-Germain de Rimouski. L'évêque de Rimouski allait encore plus loin et il demandait que les missionnaires de ce vicariat desservent en outre les Blancs de la Côte-Nord du fleuve Saint-Laurent à cause des grandes difficultés de communications entre les deux rives. Les Oblats qui avaient refusé la desserte des Blancs s'occupaient cependant des Montagnais avec "un zèle digne de louanges" ${ }^{1}$

La lettre de Mgr Langevin resta sans réponse. Quelque temps plus tard il envoyait une copie de sa lettre du mois de septembre au cardinal, car, disait-il, il craignait un naufrage possible ${ }^{2}$.

En février 1870, les évêques préparaient un Mémoire sur l'érection projetée d'un vicariat apostolique au sud-est et à l'est

* Ce travail fait suite à notre étude parue dans la Revue de l'Université d'Ottawa, 30 (1960): 57-81 sous le titre de "Projets de circonscriptions ecclésiastiques: vicariat apostolique pour le nord".

1 Archevêché de Rimouski, Registre des Lettres, B, 70.

2 Ibidem, 110. 
de la baie d'Hudson. Après avoir précisé que le vicariat, outre ce territoire, comprendrait aussi une partie des diocèses de Québec, de Trois-Rivières et d'Ottawa, le mémoire précisait:

Ils [les évêques soussignés] sont d'avis que cette œuvre devrait être confiée à une congrégation religieuse, parce qu'il n'y a qu'une telle cong $^{\mathrm{n}}$ qui pourrait l'organiser et le perpétuer. On en voit une preuve frappante dans les missions du Nord Ouest qui n'ont commencé à se développer que depuis qu'elles ont été confiées aux Pères Oblats.

Ils sont enfin d'opinion que la Congr ${ }^{\mathrm{n}}$ des Oblats de Marie Immaculée est celle qui devrait s'en charger et ce pour plusieurs raisons:

$1^{\circ}$ Parce que ces religieux font déjà les missions dans le territoire en question, aussi bien que dans le reste du Nord de l'Amérique.

$2^{\circ}$ Parce qu'ils connaissent déjà la langue et les habitudes des sauvages dispersés dans cet immense territoire, et qu'ils sont déjà accoutumés à les parcourir en tous sens.

$3^{\circ}$ Parce qu'ils ont bien réussi jusqu'à présent dans cette œuvre difficile.

Comme ils ont déjà deux résidences dans ce territoire Temiskamingue et Betsiamites, il leur suffirait d'ici à quelques années, d'y faire deux nouveaux établissements, l'un à l'est, l'autre à l'ouest de manière que cette œuvre ne leur demanderait qu'un petit nombre de sujets de plus. D'ailleurs leur noviciat à Montréal renferme déjà plusieurs sujets canadiens et les Ev. de la Province seront heureux de continuer à pourvoir de semblables vocations.

Jos. Eug. Ev. d'Ottawa

L.F. Ev. d'Arhedon

Jean Ev. de S.G. de Rimouski.

A la suite de ce Mémoire on trouve, dans la copie de l'archevêché de Québec, des notes inscrites par l'archevêque de Québec. Ce dernier prétend qu'il n'est pas nécessaire d'établir un vicariat apostolique pour la Côte-Nord, malgré les difficultés de desservir à la fois la côte du fleuve et la région située au-delà de la hauteur des terres. Et il ajoute: 
C'est ce que je sais, et ce que je puis affirmer avec connaissance de cause. Depuis trois ans j'ai réussi à envoyer à grands frais, un courageux père Oblat à la première tribu que l'on rencontre au delà de la hauteur des terres, et ses rapports montrent que ce n'est qu'avec des efforts et des peines infinies qu'il a pu y parvenir. Il lui a fallu toute une saison pour y aller et pour en revenir, et encore avec ces trois voyages n'a-t-il pu arriver à temps pour rencontrer les sauvages qu'une seule fois, grâce à Dieu, il a pu en baptiser un grand nombre.

Les Evêques qui ont écrit et signé le mémoire ci-dessus ignoraient ces circonstances. Les connaissant, je me fais un devoir de les exposer à l'Em. préfet de la Propagande et de rectifier ainsi les limites à donner au futur vicariat apostolique qu'il désire établir au nord de la province de Québec.

C.F. Arch. de Québec ${ }^{3}$

L'archevêque affirmait également que Rimouski pouvait continuer à diriger ces missions.

Le 4 février, de Rome où il se trouve, Mgr Guigues s'adresse à l'archevêque de Québec:

Selon votre désir, je réponds aux deux demandes que Votre Grandeur m'a adressées : 1) Le travail sur le vicariat à créer dans les régions du Nord de la Province de Québec, et confié à Mgr Laflèche, Langevin et moi est terminé, mais avant de le remettre à Son Eminence le Cardinal Barnabo, nous tenons à ce qu'il soit soumis à l'examen des Evêques de la Province, d'autant plus que, plusieurs doivent concourir à lui fournir les ressources pécuniaires.

2) Quant au projet de la division du diocèse d'Ottawa, je crois qu'il doit être ajourné jusqu'à ce que celui du vicariat ait reçu une solution. Votre Grandeur n'ignore pas qu'une partie du vicariat dont nous proposons l'érection au Saint-Siège, est prise de mon diocèse. Si le St-Siège donne suite à notre demande au sujet du vicariat, il nous sera facile alors de tracer les limites qu'il convient que les deux nouveaux diocèses conservent, et de donner

3 Archevêché de Québec, dossier R.R. III - 170. 
à chacun ce qui lui est nécessaire pour se fonder et se développer. Si au contraire, le vicariat ne peut être érigé ou pour une raison ou pour une autre, dans la division de mon diocèse qui sera proposée, il sera nécessaire d'examiner dans quelle partie devra se trouver la région des missions sauvages ${ }^{4}$.

Dans une lettre au cardinal préfet, le 9 février, Mgr Turgeon précise les limites et énumère les difficultés du territoire. Il termine:

Je prends aussi la liberté d'informer V.E. que le Supérieur des Oblats est à Rome en ce moment et qu'il doit en partir bientôt. Il consentirait probablement à se charger du susdit Vt Apostolique, si vous vouliez bien user de votre haute influence pour l'y engager. Or, comme j'ai déjà eu l'occasion de vous écrire, je ne connais point d'autres congrégations religieuses qui puissent remplir cette mission difficile aussi bien que celle des Oblats de Marie Immaculée ${ }^{5}$.

Le cardinal Barnabo ne dut pas manquer de voir le père Joseph Fabre, supérieur général, ou du moins de lui écrire. Le 18 mars déjà, le père Pierre Aubert, assistant général, écrivait en effet à Montréal. La question du vicariat demandé aux Oblats avait été remise sur le tapis à la demande du cardinal Barnabo qui avait appris le projet des évêques du Canada de demander l'érection d'un vicariat au nord de leur province et qui, ne voyant rien venir, leur avait demandé ce qu'était devenu ce projet. Les évêques avaient alors formulé leur demande. Nosseigneurs Guigues, Langevin et Laflèche avaient été chargés par leurs confrères de rédiger le projet, mais cela s'était fait singulièrement. Ils avaient profité du départ de Mgr Taché pour lui laisser toute la partie de son diocèse dans la baie James et on y ajoutait tout ce qui embarrassait les évêques d'Ottawa et de Rimouski ${ }^{6}$. Ce projet, remis à la maison générale, le supérieur avait chargé

4 Ibidem, dossier D. O. I - 128.

5 Archevêché de Québec.

6 On a vu dans la lettre de Mgr Guigues à Mgr Turgeon que l'on voulait consulter les évêques avant de procéder. Mgr Taché pouvait alors exprimer son point de vue. Le père Aubert qui avait lutté fort contre l'érection du vicariat bataillera avec la même ardeur contre les préfectures. 
le père Aubert d'écrire un rapport pour le détruire. Il l'avait fait, disait-il, en développant ces trois idées: 1. Le vicariat est impossible, parce qu'il ne peut être visité, ni même gouverné par un seul vicaire apostolique. 2. Lors même qu'il serait possible, il n'est pas nécessaire puisque l'état présent est préférable à celui qu'on propose. 3. Et lors même qu'il serait nécessaire, les Oblats ne peuvent s'en charger, faute de sujets pour le desservir. Le père Aubert affirmait qu'avant cela il avait dit sa façon de penser aux évêques du Canada. L'archevêque de Québec avait affirmé au cardinal Barnabo que, pour faire accepter ce vicariat par les Oblats, il fallait d'abord gagner le père Aubert. Il aurait cependant voulu qu'on lui en parlât, car il aurait appris des choses ignorées. "S'il lit mon mémoire, disait-il, il verra que nous avons de bonnes raisons pour refuser ${ }^{7}$."

Les archives générales conservent un document du père Aubert au père Fabre, daté de 1870 et intitulé Vicariat apostolique au sud et à l'est de la Baie d'Hudson, projet rédigé et signé par Mgr les Ev. d'Ottawa, D'anthédon, et de St-Germain de Rimouski. Le document est très long et procède par questions et réponses.

1. Le vicariat projeté est-il possible ?

2. Peut-il être visité et même dirigé par un seul vicaire apostolique?

3. A-t-il des revenus, non seulement pour entretenir les missions qui y existent déjà, mais encore pour subvenir aux établissements qui pourront se fonder plus tard, afin de pouvoir évangéliser les quelques Indiens qui l'habitent?

Le père répondait ensuite à ces interrogations.

1. Il suffit de jeter les yeux sur la carte du pays et d'en connaître la topographie pour se convaincre qu'un seul vicaire apostolique ne peut ni visiter ni même diriger toutes les missions de son vicariat. Le vicariat se compose de quatre parties bien distinctes.

7 Archives provinciales O.M.I., dossier Administration générale. 
a) Le bassin du Haut Ottawa compris entre les eaux de la rivière Mattawa et la hauteur des terres qui séparent le Canada du territoire de la baie d'Hudson.

b) Une partie du bassin de la baie James, c'est-à-dire toute la partie qui appartient au diocèse d'Ottawa. Le père faisait ici une observation. Dans la division proposée, on était tombé dans les mêmes erreurs que celles commises lors de la création du diocèse d'Ottawa. Au lieu de donner à ce diocèse tout le bassin de la baie James pour limites, on avait tiré une ligne géographique de la rivière Severn au cap Henriette, de sorte qu'on l'avait coupé en deux et on laissait à Monseigneur de SaintBoniface les parties qu'il ne pouvait ni visiter ni faire visiter, tandis que l'évêque d'Ottawa pouvait facilement les visiter et que les Indiens qu'il fallait évangéliser dans cette région étaient en réalité les diocésains de l'évêque de Saint-Boniface. On maintenait cette erreur pour ne pas paraître donner au vicariat projeté une trop grande étendue, mais en réalité, si le projet s'effectuait, le nouveau vicariat n'aurait pas même la charge des Indiens qui habitaient cette partie du bassin de la baie James. Le père pouvait ajouter encore bien des réflexions, mais il devait se borner et revenir sur les divisions qu'il avait commencé à énumérer.

c) La troisième partie comprend cette région désolée qui porte le nom de Labrador, qui s'étend du bassin de la baie James jusqu'à la Baie-des-Esquimaux, ayant pour limites à l'ouest la baie d'Hudson, au nord et au nord-est, le détroit d'Hudson et à l'ouest l'océan Atlantique et au sud le Canada.

d) La quatrième partie comprend le pays situé sur le côté nord du golfe Saint-Laurent de la rivière Betsiamites jusqu'à la Baie-des-Esquimaux, y compris le territoire arrosé par la rivière "Meschickenau" ou North West River, contenant en superficie environ 855,000 milles carrés.

La difficulté ne provenait pas de l'immensité de ce vicariat, mais de sa configuration qui faisait que, même relié géographiquement en chacune de ses parties, ces parties se trouvaient en réalité séparées par des obstacles insurmontables, lesquels se rencontraient dans la troisième région désolée comprise entre 
la baie d'Hudson et le district du même nom, région aussi étendue qu'un royaume. L'auteur du mémoire ajoutait ensuite quelques remarques supplémentaires sur la dimension du vicariat.

Trois points seulement pouvaient servir de résidence au vicaire apostolique: Témiscamingue, Betsiamites et la Baie-desEsquimaux, qui ne possédait aucune résidence de missionnaire. Or quel que fût celui de ces postes que choisît le vicaire apostolique, il ne pourrait visiter les missions, et ne diriger qu'une partie des missionnaires sous ses ordres. S'il se fixait à Témiscamingue, il pourrait sans doute visiter et administrer toute la partie de son vicariat dans le Haut-Canada et la baie James, mais s'il voulait visiter les missions situées sur la Côte-Nord du golfe Saint-Laurent, il devrait traverser dans toute leur largeur les diocèses d'Ottawa, de Montréal, de Trois-Rivières, de Québec et encore parcourir trois cents lieues hors de son vicariat et difficilement.

Si le vicaire choisissait Betsiamites pour sa résidence, ou encore la Baie-des-Esquimaux, il éprouverait les mêmes difficultés pour visiter le nord du Témiscamingue. S'il se fixait à la Baie-des-Esquimaux, il lui faudrait faire un trajet de plus de cinq cents lieues. Un vicaire apostolique ne pourrait donc ni visiter ni diriger le vicariat.

2. Le père Aubert affirme ensuite qu'il n'insistera pas sur la question financière, qui ne laisse pas cependant d'avoir son importance. Le vicariat ne fournira jamais par lui-même les ressources pour subvenir à l'entretien des missionnaires et aux frais qu'entraînent les voyages qu'ils devront nécessairement faire pour évangéliser les peuplades confiées à leurs soins. Les évêques du Canada assurent, il est vrai, une somme annuelle de $8000 \mathrm{frs}$, mais pour trois ans seulement et ensuite le vicariat sera à la charge exclusive de la Propagation de la Foi dont les secours, par le passé, n'ont point suffi sans l'aide de la Compagnie de la Baie d'Hudson et la générosité de quelques évêques du Canada. Tous ces secours sont précaires et n'ont pu suffire que grâce aux privations que se sont imposées les missionnaires chargés des missions déjà établies. Et quand le vicariat sera établi, trouvera-t-on de plus grandes ressources ? C'est fort 
douteux. Quelle sera alors la situation d'un vicaire apostolique qui ne pourra fournir à ses missionnaires ce qui leur est même indispensable pour vivre ? Ce n'est sans doute qu'une hypothèse, mais elle est loin d'être chimérique.

En résumé: le projet soumis par les évêques du Canada propose un vicariat qui ne pourra être visité, ni administré par un seul vicaire apostolique et qui ne garantit aucune ressource certaine pour subvenir aux besoins des missionnaires. Ce vicariat semble donc impossible. On doit déduire de là aussi que le vicariat n'est point nécessaire, ce qui cependant demande à être démontré, par cela que l'état actuel est préférable à celui que l'on veut créer.

Toute la partie détachée d'Ottawa peut être dirigée et même visitée par l'évêque d'Ottawa et on doit en dire autant de la partie séparée du diocèse de Rimouski; dès lors l'état actuel des choses est préférable à celui qu'on veut lui substituer. Il est vrai que ces contrées sont confiées aux soins des Oblats de Marie-Immaculée et que les évêques se reposent entièrement sur eux du soin de diriger et de faire ces missions. On pourrait croire dès lors qu'il importe peu que ces contrées fassent partie de leurs diocèses, puisqu'ils en ont abandonné la direction aux Oblats. A cela, on répond que les missionnaires établis à Témiscamingue, comme ceux qui résident à Betsiamites, dépendent du provincial du Canada demeurant à Montréal et que ce dernier peut communiquer aussi bien avec les missionnaires qui sont au nord qu'avec ceux qui sont à l'est, mais que, par la création du vicariat, le provincial du Canada cessera par là-même d'avoir autorité sur les sujets de la congrégation répandus en ces contrées puisqu'ils passeront de plein droit sous l'autorité d'un vicaire apostolique. Si le vicaire était choisi dans les rangs de la congrégation des Oblats, dès lors l'avantage qui existe actuellement cesserait. On peut ajouter qu'il ne semble pas très avantageux d'enlever à des diocèses constitués et ayant leurs ressources propres, des parties de territoire qui ne peuvent en avoir. C'est les établir dans un état précaire et les exposer, dans le cas d'une diminution même momentanée des allocations de la Propagation de la Foi, à une ruine complète. 
3. En supposant que la création du vicariat projeté soit réalisée, les Oblats pourront-ils s'en charger ? "J'ai répondu négativement en m'appuyant sur les raisons que vous avez fait transmettre déjà, mon T.R.P., à Mgr Guigues d'après l'avis unanime de votre conseil, quand le prélat, au nom des Évêques du Canada, vous proposa d'accepter le vicariat; raisons que je dois mentionner ici." On ne peut accepter le vicariat qu'autant qu'il serait possible à la congrégation de lui fournir des ouvriers évangéliques selon le besoin des âmes; or cela n'est point possible. Les Oblats dirigent quatre vicariats dans le Nord-Ouest de l'Amérique septentrionale. Chaque courrier apporte des réclamations des vicaires apostoliques qui en ont la charge, afin d'en obtenir des sujets et on se voit forcé... "à notre grand regret de leur répondre que nous n'en avons pas". Les vocations deviennent rares et rien ne fait espérer qu'elles augmenteront. Des sujets qui arrivent, tout ce qu'on peut espérer, c'est qu'ils soient capables de remplacer dans les maisons les vides que la mort, les infirmités précoces creusent dans les rangs des missionnaires. Quand on ne peut suffire aux besoins impérieux des vicariats dont on est chargé et dont on est responsable devant Dieu et devant l'Église, serait-il prudent de se charger d'un nouveau vicariat? Le simple bon sens répond: non. "Nous tromperions la confiance que les évêques du Canada veulent bien nous témoigner, car il nous serait impossible de répondre à leur attente."

On disait sans doute que le vicariat à ériger était déjà desservi par les Oblats et qu'il suffisait pour le moment d'avoir deux nouveaux établissements. Ces deux nouveaux établissements, les Oblats ne pouvaient les faire, pas même un seul, car ils n'avaient pas un sujet de disponible. On disait encore que le noviciat du Canada fournirait des candidats pour le vicariat. On n'en doutait pas, mais quel que fût leur nombre, il ne suffirait pas à répondre aux besoins de la province et des vicariats à la charge des Oblats.

Le père Aubert terminait sur une autre remarque:

Quand on a eu le projet de proposer au SaintSiège le Vicariat Apostolique dont je viens de parler, on aurait dû en parler aux missionnaires qui sont 
sur les lieux et qui seuls sont compétents pour donner tous les renseignements indispensables en cette matière, car eux seuls connaissent bien ces contrées. En tout état de choses, je pense qu'il ne faut pas qu'on donne suite au projet sans avoir leur avis, si les raisons que je donne ne peuvent que devoir se faire rejeter ${ }^{8}$.

C'est donc un plaidoyer absolument sed contra que nous présente le père Aubert: il finira par gagner la partie, mais non sans peine. On doit le dire: c'est lui, encore plus que le père Fabre, qui est responsable de l'échec du projet.

Mais que sortirait-il des nouvelles des tractations en cours ? Le 23 mars 1870, le père Soullier écrivait au provincial:

Rien de décidé encore au sujet du Labrador. Il est cependant probable qu'au lieu d'un Vicariat apostolique, que nous repoussons, on nous offrira deux préfectures apostoliques ayant leur siège à Betsiamits et à Témiskaming. Nous attendons ${ }^{9}$.

Le 15 avril 1870, le père Vandenberghe rapportait une parole adressée à lui par Mgr Guigues à la suite du refus du vicariat apostolique: "Coûte que coûte je m'en débarrasserai". Et le père ajoutait: "Ils ne sont pas moins zélés à Québec et à Rimouski ${ }^{10}$." Le père Aubert semble se laisser aller à l'exagération en voulant à tout prix gagner la partie. Le père Flavien Durocher écrivait à Mgr Faraud, le 27 avril 1870, qu'il avait été question d'ériger un nouveau vicariat apostolique et que l'affaire avait dû être portée à Rome. "Plus on différera, plus les difficultés seront grandes. Vous le savez, les sauvages imbus de fausses maximes ne reviennent presque jamais à courber leur tête sous le joug de la foi ${ }^{11}$."

Le 6 mai 1870, Mgr Guigues informait le provincial de récentes décisions. Sur la demande du cardinal Barnabo et en réponse aux évêques, la nomination d'un vicaire apostolique s'im-

${ }^{8}$ Archives générales O.M.I., dossier Préfectures.

9 Archives provinciales O.M.I., dossier Administration générale.

10 Archives générales O.M.I., dossier Vandenberghe.

11 Ibidem, dossier Durocher, Flavien. 
posait et il était tout naturel de le prendre chez les Oblats déjà chargés de ces missions. Sur ce, le cardinal fit appeler le supérieur général. Bien des pourparlers s'ensuivirent et le père Aubert, entre autres, se montra fort récalcitrant, mais devant l'insistance du cardinal, on lui proposa plutôt la nomination de deux préfets apostoliques: l'un à l'est où missionnait le père Arnaud, l'autre à l'ouest à Témiscamingue. "Je présume que l'on donnera suite à ce projet", ${ }^{12}$ concluait Mgr Guigues. Les évêques canadiens, de passage à Rome pour le concile, avaient dû avoir des entrevues avec le cardinal Barnabo et l'aider à résoudre les difficultés présentées par le père Aubert. En fin de compte le cardinal Barnabo avertit le supérieur général que la Propagande avait érigé deux préfectures apostoliques pour le nord de l'Amérique, plutôt que le seul vicariat apostolique demandé par les évêques. Les Oblats auraient maintenant à choisir les lieux, à déterminer les limites respectives des préfectures et à proposer trois noms pour chacune d'entre elles. Les préfets étant de simples prêtres, les préfectures relèveraient de la province du Canada.

Pour aider le provincial à préparer les réponses, le père Aubert proposait de créer la préfecture du Labrador et la préfecture de la baie James. La préfecture du Labrador comprendrait les côtes septentrionales du golfe Saint-Laurent depuis le bassin de Betsiamites jusqu'à la limite du Canada, et dans le territoire de la baie d'Hudson, toute la région adjacente au nord, c'est-àdire les bassins dont les eaux se jetaient dans la baie d'Ungava et le Labrador. La préfecture de la baie James comprendrait le bassin de la rivière Ottawa à partir de Mattawa, et dans la baie d'Hudson, tout le bassin de la baie James.

Betsiamites fournirait des ressources à la préfecture du Labrador; dans celle de la baie James, le poste de Mattawa serait fort avantageux ${ }^{13}$.

Le provincial répondit aux observations du père Aubert, le 8 juin.

${ }^{12}$ Archives provinciales O.M.I., dossier Ottawa, 1ère maison, Evêché.

13 Ibidem, correspondance, I: 224-225 et archives générales O.M.I., dossier Vandenberghe. 
Je ne voulais aujourd'hui vous entretenir que des nouvelles préfectures. Peut-être avons-nous gagné quelque chose en évitant un vicariat; mais la nouvelle combinaison créera à l'administration de la province bien des embarras, surtout si les titulaires ne sont pas de facile composition. La combinaison est nouvelle chez nous et exige que l'on en examine tous les détails.

Un point capital pour ces Messieurs sont les finances, croyez-vous qu'un simple préfet aura la même influence soit auprès de la propagation de la foi, soit auprès des populations, faire de l'argent c'est un grand point.

$2^{\circ}$ Sommes-nous assurés du concours de la Propagation, car évidemment les Evêques du Canada cesseront de payer leurs allocations.

$3^{\circ}$ Croyez-vous que les évêques ne cessent aussi de payer les allocations accordées sous d'autres titres comme pour les chantiers et pour la maison de Montréal.

$4^{\circ}$ Les préfets devront ou voudront administrer les fonds de leur préfecture. Comment régler les rapports financiers entre la Province et les Préfectures?

$5^{\circ}$ Les sujets établis sous les Préfets, deviendront-ils indépendants du provincial soit pour en être retirés, soit pour changer de résidence dans la préfecture?

$6^{\circ}$ Les Préfets devront-ils traiter directement avec Rome et avec les évêques voisins ?

$7^{\circ}$ Les Préfets seront-ils en rapport immédiat avec la Propagation de la foi ?

Voilà quelques questions qu'un premier examen me suggère et sur lesquels je vous prie de réfléchir pour nous donner une direction complète. Il s'agit d'obéir au Saint-Siège et par conséquent de nous en tirer de notre mieux. Une chose aussi fort embarrassante, c'est que dans le golfe Saint-Laurent se trouvent plusieurs postes occupés par des prêtres séculiers. Déjà j'ai écrit pour avoir des informations certaines, mais que feront ces messieurs sous la nouvelle administration. Je pense que l'évêque de Rimouski les retirera: comment pourvoir à la des- 
serte de ces postes, qui exigent la présence habituelle du prêtre. Je ne sais si vous savez que l'on a commencé sur les côtes l'exploitation du fer. Je vous remercie d'avoir voulu me donner votre opinion sur la formation et l'administration des préfectures. Vous me permettrez en même temps certaines observations :

$1^{\circ}$ Les limites que vous indiquez pour les deux préfectures laissent en dehors les postes qui sont sur le Saint-Maurice. C'est peut-être avec intention que vous l'avez fait. En effet il serait bien permis de laisser au moins une petite part à la sollicitude des évêques, d'autant plus que la civilisation avance rapidement de ce côté.

$2^{\circ}$ Vous n'indiquez aucune limite certaine $d u$ côté de l'Ouest pour le Vicariat de la Baie S. James. Ce sera le moment de trancher la difficulté entre Monseigneur Guigues et Monseigneur Taché. En tous cas Monseigneur Guigues reconnaît avoir une certaine étendue sur la côte ouest de la Baie James, seulement il ne veut pas aller jusqu'à la hauteur des terres. Pour nous si mes appréciations ne sont pas fautives, je crois que le pays de l'Ouest est le seul où le missionnaire peut espérer quelque succès: sur les côtes de la mer les protestants ont eu le temps de s'établir trop solidement pour y faire du bien pendant quelque temps encore.

$3^{\circ}$ Pareillement en donnant le Matawan pour limite au sud, vous limitez la rive droite de l'Ottawa; mais sur la rive opposée le chemin reste libre jusqu'à Ottawa même. Ceci n'est qu'une rectification à faire.

$4^{\circ} \mathrm{Je}$ ferais des objections au choix que vous faites des titulaires. Au Golfe Saint-Laurent s'il s'agit uniquement de parcourir les terrains des sauvages, ce serait excellent mais diriger les postes, peut-être gouverner des prêtres séculiers, serait chose qui lui plairait bien peu, lui qui regarde comme un supplice de rester quelques jours en société. Pour l'autre préfecture, je crains que la santé du candidat ne soit un obstacle réel: un asthme très fréquent l'empêche de voyager, et puis aussi il lui manquerait peut-être certaines qualités. L'ayant vu de près et sur les lieux, j'ai été surpris de lui voir si peu l'esprit de réflexion et de combinaison. Quel nom mettre à 
la place du sien ? Je ne vois que celui du père qui connaît les missions, les langues et les pays. Celui-là nous ferait bien défaut ailleurs et de plus en le nommant nous nous créerons des embarras; il n'a aucun esprit de conciliation: poursuivant les petites misères avec une acrimonie et une persévérance qui ne permettent aucun repos et puis à cheval sur ses droits ou sur ses devoirs ou sur sa responsabilité il sacrifierait toujours les intérêts de la Congrégation à ceux de son Oeuvre.

Plus je réfléchis, cher Père, plus je suis embarrassé de la besogne que nous venez de m'imposer ${ }^{14}$.

Quant aux noms proposés, le père Aubert, dans sa lettre du 10 mai, mettait de l'avant les pères Babel et Arnaud pour le Labrador et les pères Pian et Lebret pour la baie James et, à défaut du père Lebret, le père Garin. Il demandait de choisir trois noms pour chaque préfecture et il lui semblait que le père Babel pourrait être à la tête du Labrador et le père Pian à la tête de la baie James, les autres noms n'étant là que pour la forme ${ }^{15}$.

Pour régler cette affaire des préfectures, le provincial demanda l'avis de quelques pères expérimentés. Les archives provinciales conservent à ce sujet deux lettres, l'une du père Durocher et l'autre du père Tabaret.

Le père Durocher écrivait de Saint-Sauveur de Québec, le 11 juin 1870:

J'ai reçu avant hier votre lettre du 3 juin. Les sujets que vous y indiquez sont on ne peut plus graves. Il est difficile de reculer devant l'affaire des nouvelles Préfectures, puisque déjà notre très Révérend Père Général s'est prononcé à Rome en présence de l'Archevêque et des Evêques d'Ottawa et de Rimouski. Toute la difficulté regardait le personnel, s'il faut en croire notre Archevêque. Si ce rapport est fidèle, il ne s'agirait plus que de rendre la chose le moins onéreux possible à notre Province. Ne pourrions-nous pas obtenir que les Diocèses de Québec et de Rimouski restassent avec leurs limites actuelles fixées au Nord à la hauteur des terres et

14 Archives générales O.M.I., dossier Vandenberghe.

15 Archives provinciales O.M.I., dossier Administration générale. 
dont les eaux se déchargent dans le S. Laurent, de sorte que la Préfecture de l'Est comprendrait le territoire s'étendant de la hauteur des terres jusqu'à la Baie d'Hudson du côté nord et de l'est à l'ouest de la Baie des Esquimaux jusqu'à la réserve et le Lac Mistassini ou autre lieu.

Par là nous serons déchargés de la côte Nord du S. Laurent. Vous le savez il y a sur cette côte du St Laurent, trois établissements considérables où résident des Prêtres canadiens à environ une trentaine de lieues les uns des autres et dans l'impossibilité de communiquer les uns avec les autres au moins pendant 6 mois de l'année. Ces Prêtres qui résident $1^{\circ}$ à la Rivière Moisie où se trouvent des mines de fer; $2^{\circ} \mathrm{A}$ la Pointe aux Esquimaux, six lieues en dessous de Mingan et $3^{\circ}$ à la Rivière Nataskuan. Ils n'acceptent ces postes que dans l'espoir d'être ensuite placés à leur goût et d'éviter les épreuves d'un vicariat.

S'il nous est impossible d'obtenir les limites ci-dessus désignées et qu'il fallût accepter tout le littoral du S. Laurent depuis la Rivière Portneuf jusqu'au détroit de Belle Isle, mieux vaudrait un vicariat apostolique. Un Evêque obtiendrait plus facilement des prêtres séculiers pour occuper les établissements sur la côte nord du S. Laurent.

Quant aux missions sauvages de Betsiamits, Sept Iles et Mingan, leur existence ne peut être de longue durée, surtout dans l'hypothèse que nos Pères auraient une résidence dans l'intérieur des terres. Si l'on veut conserver nos montagnais, il est dans l'intérêt de la Religion de leur offrir ailleurs des lieux où ils pourront se rendre plus facilement et $\mathrm{y}$ recevoir les secours religieux. Si nous persistons à garder ces postes où se réunissent les sauvages nous y trouverons toutes les difficultés du Sault St Louis \&c. Quant au choix du Préfet Apostolique le sujet $A$ [rnaud] est de beaucoup préférable à $\mathrm{B}$ [abel]. D'ailleurs A adore les sauvages, les attirerait plus facilement à leur résidence dans l'intérieur des terres. Il est notoire que le nouveau préfet doit être capable de traiter avec les compagnies marchandes et propre à se concilier les esprits. Le choix entre $\mathrm{A}$ et $\mathrm{B}$ se résume dans cette question: 
lequel des deux gouvernements est préférable un gouvernement doux ou un gouvernement sévère ? La question est décidée depuis longtemps.

Quant à $\mathrm{P}$ [ian] quoiqu'il soit affaibli par les travaux, il réunit plus de qualités que ceux déjà résident à Témiskaming ${ }^{16}$.

Le rapport du père Durocher est très intéressant parce que très circonstancié. Le père Tabaret, de son côté, écrivait:

Vous m'avez demandé mes observations sur un projet relatif aux deux Préfectures dont on veut que la Province se charge.

En vous envoyant aujourd'hui ces observations que vous me demandez je vous prie de les agréer comme une preuve de mon entière soumission à vos désirs.

Le Préfet de la Propagande sur la demande des Evêques du Canada désire que la Congrégation accepte deux Préfectures apostoliques qui comprendraient toutes les Missions sauvages qui aujourd'hui sont à la charge des Evêques du Canada. La Congrégation se voit dans la nécessité d'accepter ce qu'on lui propose. Soit mais il importe que nous nous fassions une idée exacte de ce que nous allons entreprendre.

Chaque Préfecture aura un territoire immense, mais c'est un territoire à jamais impropre à la culture, occupé aujourd'hui par qlq. tribus sauvages décimées chaque jour par les privations malgré les avantages que leur procure la Compagnie de la Baie d'Hudson et dont la ruine semble imminente. Si la Compagnie abandonne ses postes jamais comme tout semble le faire présumer, car on m'assure que l'année dernière les dividendes des actionnaires de la Compagnie ont été nuls et que cette année les actionnaires qui avaient coutume de toucher cinq à six cents louis sterling n'en ont reçu que quatre-vingtquatre.

Si la compagnie actuelle avec tous les avantages qu'elle possède ne peut se soutenir il n'est pas probable que une nouvelle réussisse à s'établir, le commerce des pelleteries se fera par des aventuriers ambulants et sans honneur, les Sauvages seront obli-

16 Ibidem. 
gés de se mettre plus en rapport avec les Blancs pour avoir des provisions ce qui hâtera encore leur ruine.

On peut affirmer ce me semble que l'œuvre qu'il s'agit d'entreprendre non seulement n'a pas d'avenir mais n'est dès à présent qu'une œuvre d'une importance bien moindre qu'il ne paraît et qui exige des dépenses et des sacrifices qui pourraient être utilisés plus avantageusement ailleurs, pour le bien des âmes.

Avant d'accepter, il conviendrait d'exiger des Evêques du Canada ou mieux encore de la Propagande de Rome une allocation suffisante et sûre sur les fonds de la Propagation de la Foi, puisque l'œuvre n'aura absolument aucune ressource et qu'elle nécessitera des dépenses beaucoup plus considérables que celles que font les missionnaires actuels. A mon avis ce serait se tromper que de compter sur les ressources que fourniraient les chantiers de l'Ottawa, car cet argent ainsi recueilli sera par la force des choses employé à bâtir des églises, des écoles et des presbytères dans la partie habitable que l'on réserve pour la Préfecture de la Baie d'Hudson. A cette occasion je ferai observer que nous ne pouvons pas espérer de jouir longtemps des ressources que se trouve le P. Reboul dans ses missions des chantiers. Un voyage que je viens de faire jusqu'au Joachin m'a convaincu qu'il est nécessaire de fonder plusieurs paroisses dans ces lieux. Les curés pour faire face aux charges qui leur seront imposées viendront quêter dans les chantiers et c'en est fait de la mission du P. Reboul.

Les deux listes présentées dans le rapport me paraissent bien inspirées.

La juridiction territoriale que possède un Préfet apostolique ne me paraît pas incompatible avec les devoirs de l'obéissance religieuse, de la part de ce dernier relativement aux Provinciaux du Canada. Mais les préfectures devraient avoir la pleine et entière administration de leurs ressources pécuniaires à la charge pourtant de payer à la Province du Canada la quote personnelle imposée à tous les Pères ${ }^{17}$. 
La lettre du père Tabaret est incomplète, mais elle nous fait assez comprendre les difficultés réelles et sérieuses qu'il voyait dans ces nouvelles circonscriptions ecclésiastiques. Dans une lettre du 19 juin 1870, il affirmait que les deux préfectures seraient une charge pour la province ${ }^{18}$, et il n'est pas douteux qu'il avait raison. Mais cela suffisait-il pour refuser ?

Le 11 juin 1870, le père Vandenberghe, dans une lettre au père Aubert disait préférer le père Charles Arnaud au père Louis Babel et le père Jean-Marie Pian plus capable que tous ceux qui résidaient à Témiscamingue de devenir préfet apostolique ${ }^{19}$, et six jours après il s'adressait au père Fabre:

Quant aux Préfectures, c'est une bien grosse affaire, qui me préoccupe singulièrement depuis la notification que j'en ai reçue par le Rév. Père Aubert. Puisque c'est un acte d'obéissance à l'égard du SaintSiège, j'espère que le bon Dieu nous viendra en aide; mais cette affaire nous jette dans un grand embarras. Je ne sais, Bien-Aimé Père, si c'est toujours votre intention que nous fassions partir deux sujets pour les vicariats du Nord-Ouest. Ce serait bien difficile pour nous si les préfectures devaient s'organiser d'une certaine façon. Je dois nécessairement examiner l'affaire en conseil, quoique le Conseil n'éclairera pas beaucoup les choses. Si ces préfectures doivent être combinées d'après les intentions bien connues des Evêques, nous ne pouvons pas y suffire et peut-être serait-il préférable que les Préfets nommés fussent indépendants. C'est me semblet-il, la grande difficulté à résoudre. Evidemment les Evêques se proposent de se défaire de tous les postes les plus éloignés. Comme l'Evêque de Rimouski des postes qu'il possède sur la Côte Nord du Golfe Saint-Laurent. L'archevêque de Québec de tous les postes qui sont au nord du Lac Saint-Jean et sur le Saint-Maurice. Peut-être l'Evêque d'Ottawa des postes qui sont sur le haut de l'Ottawa. Ces postes sont peuplés ou par des colons ou par des sauvages à moitié civilisés, qui peu à peu seront comme les sauvages du désert et du Sault Saint-Louis. Presque

18 Ibidem, dossier Université d'Ottawa, 1867-1869.

19 Ibidem, dossier Administration générale. 
partout il faut dès à présent ou avant peu des prêtres résidants; je signale en particulier les postes $\mathrm{du}$ golfe Saint-Laurent, dans lesquels résident trois prêtres à trente mille les uns des autres et n'ayant entre eux aucune communication pendant six mois de l'année. Si nous prenons au nom de la Province la charge de tous ces postes, nous nous trouvons devant une impossibilité absolue. Je ferais moins de difficultés sur le haut de l'Ottawa du côté de Témiskaming, parce que ce pays sera pays de Chantier pour quelque temps encore et en plaçant deux Pères à Matawan, l'on peut se tenir en repos pour longtemps.

Si les préfectures doivent être sous le contrôle de la province, il me semble que nous devons nous restreindre à la partie purement sauvage et exclure les postes sauvages rapprochés de la civilisation, auxquels les évêques pourvoiraient par des moyens spéciaux, du reste ils sont peu nombreux. Pour faire le travail que le Père Aubert m'a demandé, je me demande ce que nous devons vouloir et quel but nous pouvons atteindre. Ce soir même je pars pour Québec pour y rencontrer le Père Durocher, qui doit aller visiter les Sauvages du Lac Saint-Jean: plus que tout autre il peut fournir des renseignements sur les affaires de ce côté ${ }^{20}$.

Quelques jours plus tard, le 23 juin, le père provincial écrivait au père Aubert.

J'arrive de Québec [...] J'ai vu l'archevêque et j'en ai obtenu à peu près ce que je désirais savoir. Si l'affaire des préfectures se conclut il nous donnera ce qu'il avait souscrit il y a deux ans quand il s'agissait d'un vicariat. Ce que je voulais savoir avant tout, c'est la possibilité de nous débarrasser des postes connus sous le nom des postes du roi. Je vois avec plaisir que l'archevêque est en cela de notre avis et que ces postes doivent rester à l'Evêque de Rimouski.

Je ne sais s'il y a un temps déterminé pour présenter le rapport au sujet des préfectures, car il me serait fort difficile de réunir le conseil en ce moment;

${ }^{20}$ Le 17 juin (archives générales, O.M.I., dossier Vandenberghe). 
il faudrait surtout que je puisse voir les Pères Pian et Arnaud; mais je ne puis les rencontrer avant le mois d'Août. Toutefois je prends des notes et je commence un petit travail sur cette question, dont je veux vous entretenir plus longuement une autre fois ${ }^{21}$.

Le père Vandenberghe semble donc un peu rassuré et plus prêt à agir. Mais que pense la maison générale, et surtout le père Aubert qui dirige toute cette affaire ? Le père Aubert le dit bien clairement au provincial, le 27 juin:

Vous devez comprendre que nous ne sommes pas plus en faveur des préfectures apostoliques qu'on veut nous imposer que du Vicariat, quoique les préfectures présentent pour nous moins d'inconvénients que les Vicariats. Soyez sûr que si je puis faire échouer ce projet je ne m'y épargnerai pas; mais la Propagande $\mathrm{y}$ tient et il nous sera bien difficile d'échapper au fardeau. Je suis bien d'avis qu'avant toute acceptation définitive, on nous assure des ressources certaines, et que s'il y a possibilité nous ne prenions pour constituer les préfectures que du pays non constitué en paroisse. Seulement il est opportun de voir si pour fournir quelques revenus au missionnaire il ne serait pas bon de conserver dans les limites des préfectures du pays déjà constitué en paroisse. Vous avez pu envisager cette question sous toutes ses faces, avec l'aide du missionnaire du Labrador et de la Baie James et le secours de votre conseil vous pourrez faire un travail complet.

Vous aurez à examiner si les propositions faites par les Evêques du Canada lors de leur réunion à Toronto, quand il s'agissait d'ériger un vicariat au nord du Canada, vicariat qu'on voulait nous confier, sont maintenues, je parle de la question financière, et si les ressources qu'ils nous offrent sont suffisantes. Car dans ces sortes d'affaires les deux questions sont à examiner celle des sujets et celle de l'argent. Rien ne presse par rapport à la réponse, la Propagande aura la bonté d'attendre, je ne pense pas qu'elle veuille nous imposer de suite les deux préfectures sans que nous ayons reçu tous les docu-

21 Ibidem. 
ments qui nous sont nécessaires et qui doivent former notre jugement ${ }^{22}$.

Nous ne comprenons pas comment le père Aubert peut vouloir former son jugement quand il a déjà écrit en blanc et en noir: "Soyez sûr que si je puis faire échouer ce projet je ne m'y épargnerai pas." Il est bien décidé à ne pas voir l'établissement des préfectures.

Le provincial continue d'étudier la question et, le 15 juillet, il est prêt à donner de nouveaux éclaircissements :

Je reprends ma lettre et je réponds à ce que vous me dites sur les Préfectures. C'était bien compris, cher Père, que ces Préfectures ne sont pas votre œuvre et que nous devons les supporter. J'avais déjà préparé un travail sur la question. Je l'ai envoyé au Père Tabaret à Ottawa pour qu'il me fasse ses réflexions et me le renvoie au plus tôt. Voilà quinze jours qu'il a dû le recevoir et pas même il ne m'accuse réception: je sais qu'alors il était à Ottawa. Tout cela retarde, mais bien pis je crains d'y reconnaître de la mauvaise humeur: les réflexions de votre avant dernière lettre m'ont fait deviner des dispositions que je ne soupçonnais pas et que tout confirme depuis ce temps...

J'oublie nos Préfectures: nous nous sommes rencontrés sur plusieurs points, mais pas sur tous. Comme vous l'avez vu par mes lettres précédentes, je regarde comme chose capitale pour nous de n'avoir pas la charge des côtes du golfe Saint-Laurent et je crois que tous nos efforts doivent être dirigés pour éviter la charge de tous ces postes. Mes sentiments sont partagés à peu près par tous nos Pères et plus particulièrement par le Rév. Père Durocher qui connaît mieux le pays. Dans quinze jours je pense me trouver à Bethsiamits et voir le Père Arnaud. Je vous ai dit que déjà j'ai fait le voyage de Québec pour sonder l'archevêque (qui se trouve actuellement fort malade l'on craint) et connaître sa pensée sur cette question d'abord et ensuite au sujet de l'argent. Je vous ai dit que j'ai été satisfait. Si les évêques avaient été présents, je les aurais déjà vus ou bien

${ }^{22}$ Archives provinciales O.M.I., dossier Administration générale. 
je leur aurais écrit; mais tout se combine pour que cette affaire marche lentement: peut-être c'est une bonne chose. J'ai fait venir le Père Pian: il est ici depuis huit jours. J'ai pu causer avec lui sur toutes ces affaires et puis j'ai été on ne peut plus heureux en le voyant très bien portant. Son asthme a presque disparu. Nous pourrons compter sur lui et d'autant plus qu'il me paraît avoir gagné en maturité et en réflexion ...23

Le 29 juillet, le père Vandenberghe est toujours sans nouvelles du père Tabaret ${ }^{24}$, mais le 19 août, le provincial peut faire parvenir un long rapport sur les préfectures. S'étant rendu à Betsiamites, écrit-il au père Fabre, il a pu obtenir sur ces contrées des renseignements précieux qui l'aideront à arranger l'affaire des préfectures. En attendant un rapport complet dont le projet est toujours entre les mains du père Tabaret, le provincial envoie un sommaire de ses réflexions, sous le titre: Quelques notes sur la résidence de Bethsiamits et la Préfecture du Labrador.

I. Résidence de Bethsiamits. - Les Pères Arnaud et Babel ont chacun leur mérite: le premier bien affable possède toujours les sympathies des sauvages comme des blancs. L'autre énergique mais sauvage ne peut travailler que seul. Je crois que le Père Babel a eu une entente avec l'archevêque et s'attend à être nommé préfet.

La résidence se trouve à 75 lieues de Québec, au $49 \mathrm{e}$ de latitude. Les Pères y possèdent une charmante petite église et deux maisons spacieuses dont l'une est convertie en musée et l'autre donne six belles chambres à coucher avec cuisine et réfectoire et dépendances. Les terres sont bonnes: il y a dix vaches, vingt moutons, trois chevaux: trente arpents de terre cultivée, outre deux autres fermes à quelque distance. La propriété est aux sauvages: en mission ils sont 600 à 700 en nombre. La terre est réservée par le gouvernement sur un côté de la Rivière et l'autre côté sera propriété de la Compagnie de la Baie d'Hudson qui a intérêt à empêcher les blancs de

${ }^{23}$ Archives générales O.M.I., dossier Vandenberghe.

$24 \mathrm{Au}$ père Aubert (ibidem). 
s'établir: de sorte que pour longtemps les sauvages y demeureront maitres. A quatre lieues de là, à Saint Jérémie ancienne mission, les sauvages ont une propriété d'un mille carré et cette propriété a été passée aux Pères Oblats. Reste à savoir ce que vaut ce titre de propriété.

Les Pères reçoivent 500 pi. du gouvernement et le Père Babel 600 pi. de la Propagation de la foi. Ils réalisent en argent 1400 pi. et leurs dépenses non comprises la côte provinciale sont de 1000 pi. en argent.

Le climat est rigoureux et froid, mais salubre. Il y fait bon dans la saison des chaleurs. Le golfe y a seize lieues de largeur et l'on ne peut traverser à Rimouski qu'avec des embarcations légères: la traversée est donc incertaine et pendant l'hiver pour passer à Rimousky il faut faire le tour par Québec.

II. Missions des Pères Oblats de Bethsiamits. - Le diocèse de Rimousky sur la côte Nord du golfe s'étend depuis la rivière Portneuf qui se trouve à dix lieues en amont de Bethsiamits, jusqu'au delà du Grand Sablon vis-à-vis le détroit de Belle Isle et comprend une étendue d'à peu près deux cent cinquante lieues, sur une profondeur de quatre-vingts lieues à peu près, c'est-à-dire, jusqu'à la hauteur des terres. Nos Pères ont juridiction directe depuis Portneuf jusqu'à Godbout, qui se trouve à quinze lieues plus bas que Bethsiamits. De plus ils ont juridiction indirecte sur tous les sauvages de la côte. Sur leur territoire ils ne comptent que 33 familles canadiennes; mais les sauvages de toute la côte sont au nombre de deux mille. Le recensement accuse une population de 2150 âmes. Les Canadiens ou plutôt les Acadiens sont plus nombreux en bas du fleuve et forment des établissements assez importants:

$1^{\circ}$ A Moisie 30 lieues de Bethsiamits. L'on y exploite des mines de fer. L'on y compte 300 canadiens mais pour un temps seulement. Le prêtre qui y réside est entretenu par la Société qui exploite les mines.

$2^{\circ}$ Pointe aux Esquimaux. Etablissement de pêcheurs acadiens. C'est une mission bien établie à six lieues de Mingan et à 60 lieues de Bethsiamits. 
Mingan est le poste sauvage. Le prêtre de la Pointe des Esquimaux se suffit à peu près.

$3^{\circ}$ Nataskuan est encore à 25 lieues plus bas que la mission précédente et se trouve à 85 lieues de Bethsiamits. Cette mission est composée de sauvages et d'acadiens; mais elle est loin de suffire et le prêtre est soutenu par Rimouski.

Outre ces missions il y a deux postes de moindre importance; mais l'on dit qu'au Grand Sablon il y aurait opportunité d'avoir un prêtre résident. L'on dit aussi que proche du Grand Sablon il existe une tribu sauvage appelée Pieds-Noirs qui est bien disposée pour la religion.

La population totale de la côte ne dépasse pas quatre mille âmes, blancs et sauvages. Jusqu'à Mingan on ne voyage en hiver qu'à la raquette: plus au Nord on peut se servir de traînes à chiens.

III. Labrador. Les pays en dehors du Canada qui doivent faire partie de la Préfecture Apostolique forment la presqu'île qui se trouve entre la Baie d'Hudson, le Détroit d'Hudson et l'Océan. Cette contrée se partage en deux parties: celle de l'est ou le Labrador proprement dit et qui s'étend jusqu'à la hauteur des terres appartient au Gouvernement de Terreneuve et spirituellement au diocèse de Havre de Grâce. L'autre partie celle baignée par la Baie d'Hudson était dans les domaines de la Compagnie de la Baie d'Hudson, dont les droits ont passé au gouvernement canadien. Sur ces immenses pays la Compagnie de la Baie d'Hudson ne paraît bien établie que dans le district de Rupert sur la Baie de James. Partout ailleurs elle a continuellement changé ses positions, les supprimant et les rétablissant sans cesse. Je ne pense pas que la population totale dépasse 1500 âmes. Je ne connais de catholiques que 400 Naskapis baptisés par le Père Babel. Je doute de la possibilité de s'établir sérieusement dans ce pays: peut-être que le Père Babel au retour de son excursion pourra nous renseigner plus complètement. Par malheur le district de Rupert est déjà occupé par les Protestants, de même aussi les côtes de l'Océan sont occupées par les frères Moraves au Nord et par les Ecossais à la Baie des Esquimaux, (Ne confondez pas avec la Pointe des Esquimaux) où se trouve le poste de Rigoulette. 
IV. Préfecture du Labrador. Il me semble que pour former cette Préfecture nous n'avons que trois plans.

$1^{\circ}$ Abandonner Bethsiamits et le service des côtes pour transporter nos missionnaires au Labrador. Ce plan offre bien des inconvénients; d'abord l'on ne sait où s'établir et l'on ignore si un établissement est possible: en tout cas cet établissement serait dans un isolement complet durant la majeure partie de l'année; jamais il n'y aurait des ressources locales. Ensuite l'abandon des sauvages de la côte ne serait peut-être pas toléré: je regretterais notre position de Bethsiamits.

$2^{\circ}$ Conserver la mission de Bethsiamits dans les conditions actuelles sous l'autorité de l'Evêque de Rimouski et nommer un préfet pour le Labrador. Ce plan nous dispenserait de nous occuper des postes canadiens et acadiens de la côte. Ce serait le statu quo avec la différence que le Préfet aurait juridiction directe dans son district. Ce plan me sourirait si alors nous pouvions espérer les subventions de la Propagation de la Foi: l'évêque de Rimouski qui déjà dès cet automne voulait retirer ses prêtres agirait sans cesse contre nous: les Pères ne sont pas en très bonne harmonie avec lui.

$3^{\circ}$ La position serait plus franche et plus nette en comprenant les côtes du golfe dans la Préfecture. La Congrégation pourrait s'arranger comme elle veut. La difficulté est de trouver des sujets. D'après le Rév. Père Arnaud il suffirait d'avoir à la Pointe des Esquimaux près de Mingan une résidence de trois Pères et l'on pourrait supprimer les autres postes: dans quelques années une autre résidence serait nécessaire au Blanc Sablon. Je crois que toute la préfecture pourrait être administrée avec huit ou dix Pères et pour le moment il en faudrait cinq ou six.

Quant aux ressources les évêques que j'ai vus consentent à payer leur souscription pour trois ans. Elle donne 2000 pi. par an. C'est à nous d'aviser ensuite pour l'avenir.

J'ai vu Monseigneur de Rimouski qui croyait l'affaire terminée. J'ai dit à Sa Grandeur que dans le cas où Rome comprenne la côte Nord dans la Pré- 
fecture, nous ne pourrions pas remplacer ses prêtres avant un an.

Je communiquerai cette lettre aux membres du Conseil. J'espère que nous pourrons nous réunir au commencement de septembre ${ }^{25}$.

Le rapport du père Vandenberghe au père Tabaret a finalement été perdu, ce qui contrarie beaucoup le provincial, mais il promet au père Aubert de lui envoyer sous peu un rapport complet sur les préfectures ${ }^{26}$.

A Paris, le père Aubert n'est pas facilement converti. Sa lettre du 7 septembre au père provincial, le montre bien clairement. Attendons et laissons mourir l'affaire, semble son mot d'ordre:

Quant aux préfectures apostoliques du Labrador et de la Baie James, je crois que le meilleur pour le moment pour nous c'est d'attendre, car il est plus que probable que la Société de la foi de Paris Lyon ne pourra rien donner d'ici à plusieurs années. Mais cela ne doit pas vous empêcher d'étudier la question, seulement ne vous pressez pas de nous envoyer votre projet ${ }^{27}$.

Il y avait probablement des avantages à ce retard. Dans le cas où Rome aurait insisté de nouveau, on aurait pu dire qu'on attendait toujours des renseignements importants du Canada. Le père Aubert n'avait-il pas dit déjà au provincial: "la Propagande aura la bonté d'attendre ${ }^{28}$." Ce n'était certainement pas une obéissance prompte que le père Aubert pratiquait en l'occurrence.

Le Conseil provincial, en qui le père Vandenberghe ne semblait pas avoir beaucoup confiance, finit par se réunir, les 28 et 29 septembre 1870 . On y lit dans les minutes que le père provincial fit part aux membres du Conseil de la réception d'une lettre du père Aubert, en date du 10 mai, annonçant que la Sacrée Congrégation de la Propagande voulait confier deux préfectures aux Oblats. A cette occasion, le père provincial, dut présenter à son

25 Archives générales O.M.I., dossier Vandenberghe.

$26 \mathrm{Au}$ père Aubert, le 20 septembre 1870 (ibidem).

27 Archives provinciales O.M.I., dossier Administration générale.

28 Le 27 juin (ibidem). 
Conseil le second rapport sur les préfectures, dont il dit au père Fabre, le 16 septembre: "J'ai terminé mon second rapport sur l'affaire des préfectures apostoliques. J'en avais fait un premier et je l'avais envoyé au Rév. Père Tabaret qui l'a égaré ${ }^{29}$."

Le 12 janvier 1871, le Père Fabre écrivait au Père Vandenberghe lui demandant probablement des renseignements sur l'affaire des préfectures, car ce dernier répond, le 13 février:

Aussitôt votre lettre reçue je me suis mis en devoir de vous expédier le petit travail que j'avais préparé sur les préfectures apostoliques. Je vous l'envoie par la malle des Etats Unis, pour gagner quelques jours. ...

Je redoute toujours que la préfecture du Labrador ne nous embarrasse beaucoup. Je sais que l'évêque de Rimouski fera tout en son pouvoir pour jeter tout le fardeau sur nos épaules, et, pour vrai dire, il a raison; mais pour nous c'est une charge impossible. Si la question pouvait être bien expliquée à la Propagande, peut-être qu'elle serait décidée en notre faveur, mais pourvu que de son côté Mgr Langevin ne fasse agir efficacement pour lui. Je crois aussi que le défunt archevêque de Québec s'était entendu avec le $\mathrm{P}$. Babel quant à la dignité de préfet apostolique: évidemment, celui-ci s'y attend chez-nous cependant tout le monde serait surpris. Ce que je vois de plus clair en toutes ces affaires, c'est que tous les évêques, Mgr Guigues plus que les autres veulent faire peser sur la Congrégation autant d'embarras que possible. L'on dirait même que la bâtisse du noviciat les offusque. Ah ! ils ont de l'argent disent-ils. Ils peuvent faire leurs affaires, ces Oblats ! ! [ ...] Ce qui me chagrine le plus, c'est que l'évêque de Montréal ne nous a pas payé cette année les allocations pour les chantiers et les missions de la Baie d'Hudson. C'est une perte nette de 400 louis et plus, il faut que de son côté Mgr Guigues nous enlève 200 louis. L'on dirait que tous veulent s'acharner contre nous précisément au moment où ils nous imposent des charges nouvelles. De notre côté, je voudrais procéder avec prudence et lenteur. Quand les préfectures seront décrétées, avant d'en prendre 
la direction, je me propose d'écrire à tous les Evêques signataires en faveur du Vicariat Apostolique, afin qu'ils fassent honneur à leur souscription. Déjà l'évêque de Montréal recule. Ces préfectures nous créent de grands embarras ${ }^{30}$.

A sa lettre, le père Vandenberghe joignait son mémoire sur les préfectures.

(Nous publierons ce mémoire et la suite de l'article dans le prochain numéro).

Institut d'Histoire,

Gaston CARRIÈre, o.m.i.

Université d'Ottawa.

On nous prie d'annoncer:

\section{SECOND COURSE}

in

\section{ARCHIVAL PRINCIPLES AND ADMINISTRATION}

Carleton University, in co-operation with the Public Archives of Canada and the Archives Section of the Canadian Historical Association, hopes to offer a course in basic archival techniques, with special attention to archival problems peculiar to Canada, in June, 1964.

It is planned that the course will be co-ordinated by an archivist from the Public Archives of Canada, who will call upon specialists from federal, provincial and private archival institutions in Canada.

The course will include both formal and practical work, with an opportunity for students to concentrate their studies in either the field of archives or of record management

The length of the course will be approximately four weeks, the exact dates to be announced early in 1964. Tuition fees will be $\$ 85$ per student. Living accommodation and meals, at additional cost, will be available in the Men's Residences of Carleton University.

Further information and application forms will be available, early in 1964, Dept. of History, Carleton University, Ottawa. 